\title{
Development of the Neurorobotic Mouse
}

\author{
Peer Lucas $^{1}$, Satoshi Oota ${ }^{2}$, Jörg Conradt ${ }^{3}$ and Alois Knoll ${ }^{1}$
}

\begin{abstract}
In this paper we describe the NeuroRobotic Mouse (NeRmo) a low-cost, modular bio mimetic robot, mimicking the actuation and walking behaviour of a common mouse (Mus musculus). This latest version has 13 Degrees of Freedom with 21 tendon driven joints and can be controlled in both open and closed loop. It is capable of different gaits as well as keeping the body upright when in a sitting position. The robot includes joint position sensors, pressure sensors on the soles of the feet as well as two cameras in the head. As the design of this robotic platform was inspired by detailed observations of the biomechanics of mice and rats, it can be used in motion research using animal data as am element of comparison, or even as actuation input.
\end{abstract}

\section{INTRODUCTION}

Quadrupedal mammals are well adapted to move in unstructured environments using stable gaits, essential for hunting prey or escaping predators while using very little energy [1]. These two points are, among the others, motivating current robotics research into quadruped robots. Use Cases have been demonstrated for industry inspection [2], but also emergency response, security and parcel delivery [3].

Robots mimicking animals can be introduced into the same environments as the real animals and not only interact with them, as done in [4] or teaching them new motions [5]; they could also potentially provide insights into the control mechanisms needed to create the same motions and actions as the biological counterpart.

In neurosciences, rodents are often used for model organisms to mimic human biological traits; mainly because of affordability of the species as genetic and physiological resources. Especially laboratory mice have a long-standing history as a genetic tool [6]. In the Human Brain Project, we heavily rely on the mouse resource to obtain multimodal data of the brain.

Regarding the embodiment of the brain simulation, as a consequence, it is a natural approach to employ the virtual mouse body [7] as a computational simulation model. In the meantime, physical simulations [8] with a mouse robot can play a pivotal role to compensate inevitable limitations of computational simulations [9], [10]: e.g., contact interactions [11], elastic attributes [10], and unmodelable environmental factors. The mouse robot is also useful to measure internal

\footnotetext{
${ }^{1}$ Peer Lucas and Alois Knoll are with the Chair of Robotics, Artificial Intelligence and Real-time Systems at TUM, Munich, Germany.

${ }^{2}$ Satoshi Oota is with the Center for Advanced Photonics, The National Institute of Physical and Chemical Research, RIKEN.

${ }^{3}$ Jörg Conradt is with the Division of Computational Science and Technology at KTH, Sweden

The research leading to these results has received funding from the European Union Horizon 2020 program under grant agreement No. 785907 (Human Brain Project SGA2)
}

states (e.g., tension of muscle tendons and joint torques) during motions, which is hardly measurable by using live animals [12]. The significance of the mouse robot resides in the data assimilation [13] with the computational and physical simulations, as well as the multimodal data obtained from live animals.

To do so, the mouse robot requires the following minimal set of features:

- Flexible and functional backbones: Any terrestrial vertebrates have great functionality in the vertebra musculoskeletal system [14], which we had often overlooked in robotics. The feature is one of the evolutionary consequences that any terrestrial animals have marine vertebrates as their ancestors. As a result, many of the complex motor functions are inherently coordinated by the flexible trunk (axial parts of the body) [15], [16].

- 2. Biologically relevant extremities with a small number of parts: The anatomical structure of vertebrates is evolutionarily conserved [17], and mouse has almost the same complexity as human despite the size differences [18]: Note that the two species have nearly the same number of genes [19] (which are a blueprint of the biological system). The extremities should have a sufficient degree of freedom that makes it possible to give biologically relevant motions with a small number of parts.

- A functional tail: Mechanically speaking, the mouse tail has a significant role in keeping balance [20] as well as a particular dexterity with its flexible motions [21]. At least, the first condition is required.

- A minimum sensory system: Mouse behaviors rely on visual perception as well as olfactory and tactile perception with whiskers [22]. At least we should implement one of the sensory systems.

- Musculotendon actuators that roughly mimic the biological system: Since the purpose of this project is to perform physical simulation, the robot should have musculotendon actuators innervated by spike trains fed from an external brain model.

Here we developed a mouse robot that satisfies the above necessary conditions. In this paper, we describe the detailed specificity of the robot and discuss its potential applications for robotics and life sciences.

\section{Preliminary Work}

This project was started in 2017 leading to 4 different prototypes including the final version presented in this paper. The first robot was described in a technical report [23]. It was intended to be as close as possible in appearance and 
motion range to a common mouse. However, due to size and weight constraints (mostly related to the control electronics and batteries), it ended up being more comparable to a rat. Additionally, this robotic platform was from its very first iteration expected to be low-cost, modular, simple to build and simple to use. This was seen as necessary so that it would be adopted by multiple laboratories, not only for robotics research but also for neuroscience and biology. This led to the design of a robot actuated by a combination of tendon-driven and direct actuation, to reduce weight in distal limbs and obtaining an under-actuated system, as will be shown hereafter for the legs, spine and tail. Starting with Version 1 as described in the technical report, the later versions where expanded regarding motion range, Degrees of Freedom (DOF) and sensory capabilities.

\section{A. Version 1.0}

The first version was described in detail in 2018 [23]. This was an 8 Degree of Freedom walking robot with two degrees of freedom in each leg. The Legs used a pantograph design, allowing the foot to be passively actuated together with the knee actuation. The hip was directly actuated, while the knee was actuated with a tendon-driven mechanism. The robot could walk straight forward and backward in a trotting gait. It could also turn, by generating an adapted trotting gait that used a shorter step length on the inner side of the turning circle.

\section{B. Version 2.0}

The second iteration of the robot was used to test the inclusion of lateral movement in the spine and tail, in order to better control turning during walking and to include spinal and tail motions during the straight trot to increase the gait stability. A tendon-driven actuation mechanism was designed to move the spine in the lumbar and sacral regions with one actuator, and the whole length of the tail with another actuator (Figure 1). This mechanism consisted of multiple elastic joints created within the $3 \mathrm{D}$ printed model. Two tendons, running along the side of the joints on each side, bended the joints when pulled on one side and released on the other. connecting parts between the joints ware made rigid against the actuation, to guide the wires appropriately. The joints were created by thinning out $3 \mathrm{~mm}$ long parts of the spine, making the connection thin enough for elastic deformation.

The hand-made spring system in the legs of the previous version was replaced for the hindlegs with machined parts, performing the same task to avoid discrepancies between the two legs by increasing manufacturing precision. For the forelegs, the design was changed in such a way that no machined parts were necessary and the springs could be directly attached to the 3D printed legs. Both Legs can be seen at the top of Figure 2.

The control was provided by a Teensy board together with a Bluetooth communication board. The gait generation and walking control was outsourced to a remote laptop running ROS.

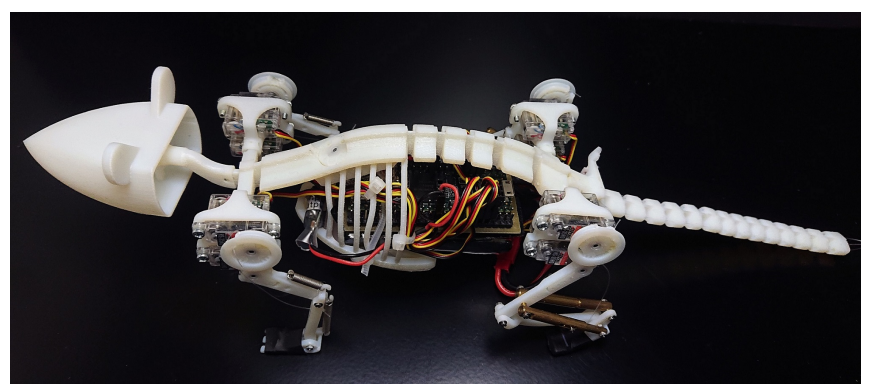

Fig. 1. NeRmo Version 2.0 with flexible spine and tail

\section{Version 2.1}

Version 2.1 used the same body and control design as V2.0, but with monobloc printed legs, with the goal of reducing the complexity for the leg design form 26 parts (V2.0) to one part. This simplification was done by replacing the mechanical joints of the previous legs with flexible hinge joints. The 3D-printed material used (PA 2200 from EOS $\mathrm{GmbH})$ is not only flexible but also elastic, meaning the springs could be replaced by an adequate joint design as well, making the flexible hinges stiff enough to create the needed extension force to support the robot. Using multiple test joints, changing material thickness, shape and width to reach the same performance as the previously designed legs, it could be shown that the simplification of the legs is possible. Both designs can be seen in Figure 2. Some drawbacks had to be noted, however. In particular, the pantograph design of the jointed legs could not be recreated with a single piece design. The solution was to fix the angle of the foot: the latter indeed caused collisions between the toes and the ground during the swing phase of the leg, and it also prevented the whole sole of the foot to contact with the ground, which is needed to generate enough friction to propel the robot when climbing up slopes.
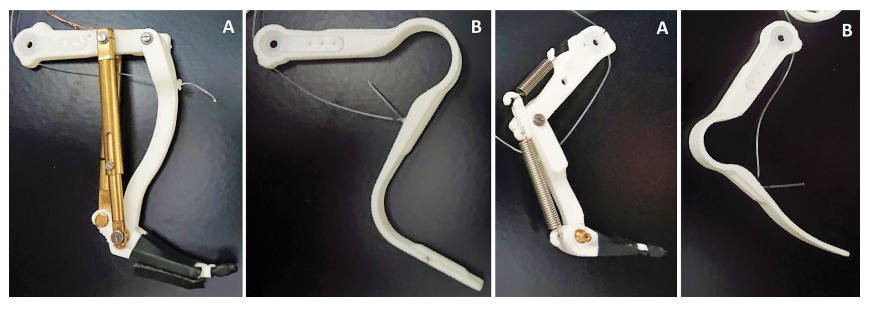

Fig. 2. NeRmo hind- and forelegs of Versions 2.0 (A) and 2.1 (B)

be created by replacing the joints with flexible hinges which are stiff enough to create the needed extension force to hold the robot

\section{Version 3.0}

The third version of the mouse implemented an additional DOF for the lumbar flexion of the spine. The flexion of the Spine can often be seen in mice and rats as they sit 
upright to eat, and even during walking, where the spine is stretched and crouched during standing. This was realized by introducing rubber bands as antagonists on the ventral side of the spine and a servo-pulled tendon on the dorsal side as the agonist. As the joint connections proved to be too flexible, a foam cushion was introduced and glued to both sides of the joint to dampen the motion and improve the spine stability as can be seen in Figure 3. However, it could not be guaranteed, that when actuating the spine in one single axis (lateral flexion or lumbar flexion) the respective other axis would not be bend as well, which made a definable control impossible.

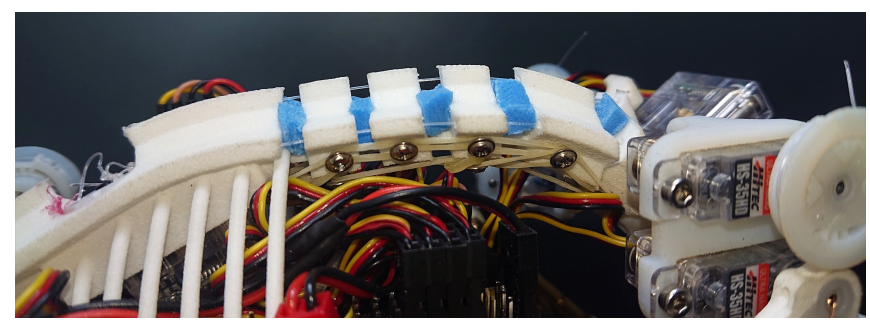

Fig. 3. NeRmo Version 3.0, Spine. The Rubber bands are the antagonists, the nylon wire on the top is the agonist.

\section{Neurorobotic Mouse}

The latest version of the NeRmo will be the last major evolution of the robot design, as it provides enough capabilities to be used as a research platform. The base characteristics can be found in Table I.

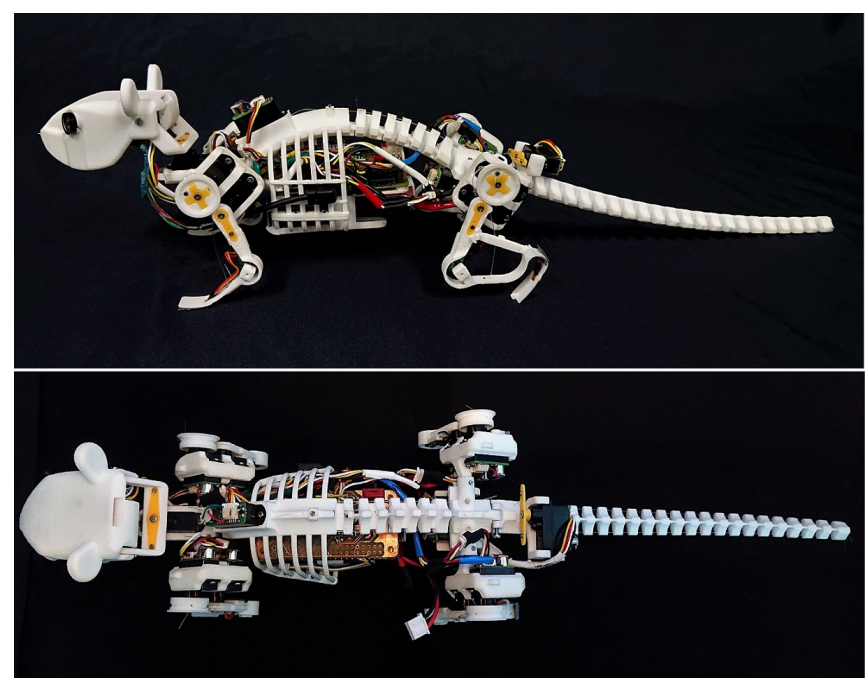

Fig. 4. NeRmo Version 4.0

The overall design can be seen in Figure 4. The core body model was redone from scratch, especially for the spine as explained for V3. The motion is now defined through alternating flexible hinges for lumbar or lateral flexion. The maximal motion is limited by fixed end stops, to prevent over-flexing of the hinges. The design was made sturdier than previous versions, especially in the rib cage area, in order to reduce mechanical failure. Overall 13 DOFs are included into this design. Two for each leg, one for the tail, two for the spine (flexion and lateral flexion) and two for the head (pan/tilt). The head itself was redesigned to allow space for the tilt servo; it also accommodates two USB cameras, a USB hub, a capacitive touch button between the ears and a touch button on the nose. The tail was adapted, to fit the biological model in length and shape with a tapered end. It is actuated for lateral flexion as the previous versions, but can also be moved passively up and down in order to enable the robot to sit upright. All tendons can now be fixed using screws instead of knots and are more precisely guided through the robot. In particular, slip-off of the wires is now prevented for the coils at the shoulders and hips, as this occurred from time to time in earlier versions. The ribcage was redesigned to allow more space for the electronics as well as simple disassembly. The battery can now be changed easily as well as charged wirelessly using a QI-standard cell phone wireless charger.

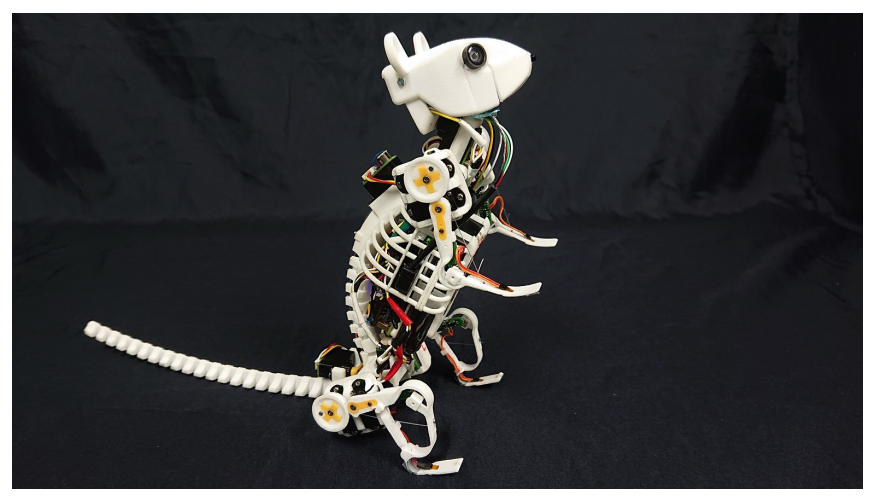

Fig. 5. NeRmo Version 4.0 in sitting position

The motion range of the legs allows for the robot to not only walk, but to also sit upright (see Figure 5) to use the fore paws to actuate levers.

TABLE I

SPECIFICATIONS

\begin{tabular}{ll}
\hline Degrees of Freedom & 13 \\
\hline Elastic Joints & 27 \\
\hline Speed & $\approx 0.3 \mathrm{~m} / \mathrm{s}$ \\
\hline Physical & $405 \mathrm{x} 91 \mathrm{x} 90[\mathrm{~mm}]$ \\
\hline Size & $117[\mathrm{~mm}]$ \\
\hline Length Scapula-pelvis & $265[\mathrm{~g}]$ \\
\hline Weight & \\
\hline Electrical & $7,4 \mathrm{~V} 1000$ mAh LiPo \\
\hline Battery & $\approx 25 \mathrm{~min}$ continuous walking \\
\hline Run Time & $2.4 \mathrm{GHz} 802.11 \mathrm{n}$ WLAN \\
\hline Connectivity & \\
\hline Sensor & Every actuator can provide \\
\hline Actuators & position and current \\
\hline Legs & Knee Angle \\
\hline Feet & Ground Pressure \\
\hline Body & IMU \\
\hline Buttons & Nose and Head \\
\hline Head & Two wide Angle HD Cameras \\
\hline Cost & $\approx € 850$ \\
\hline
\end{tabular}




\section{Sensors and control electronics}

The most significant improvement over the previous versions is that the robot now possesses proprioceptive abilities, with an IMU above the centre of gravity, joint angle sensors at the hip and knee joints, and pressure sensors on the soles of the feet. To allow this level of proprioception, multiple custom PCBs had to be created. The electronics of the servos were replaced; the new electronics have their own microcontroller, which can be addressed via single wire UART. This allows multiple servos to be daisy chained. Additionally, the position sensors were replaced with hall sensors rather than the usually used rotary potentiometers. The latter are indeed prone to mechanical failure when submitted to repetitive motions. As the hall sensor is contactless, this type of failure is averted. Moreover the motor current can be measured, thus allowing one to infer the motor force.

The legs are fitted with another PCB, including a hall sensor, to infer the angular position at the knee or elbow. An ADC converts the analog readings from the pressure sensor in the foot and sends them via the same I2C bus as the hall sensor to reduce wiring to and from the leg. The $\mathrm{I} 2 \mathrm{C}$ Bus is connected to the nearest servo motor and can be read through addressing this motor.

The main control unit is now a Raspberry Pi Zero W with WLAN and Bluetooth accessibility. A secondary control unit, the "Spine" is implemented in an adapter board for the Raspberry Pi, which handles the Single wire UART busses as well as the IMU, power management and the IO of the buttons. There are 6 UART busses on the spine, four for the legs, one for the spine, tail and head, and one for communications with the Raspberry Pi.

The Power management uses a 7,4 V, $1000 \mathrm{mAh}$ battery, which is regulated to $3 \mathrm{~V}$ for the spine and $5 \mathrm{~V}$ for the Raspberry Pi. Three Regulators to $6.3 \mathrm{~V}$ are used for the servos, with two for the left and right legs and one for the spine, tail and head servos. To charge the battery a QI-standard charger can be used. The resulting $5 \mathrm{~V}$ are transformed to charge the LIPO Battery.

\section{Control}

With a Raspberry $\mathrm{Pi}$ as the base computing platform, Raspian, a Debian-based operating system can be used to setup the robots control structure. This allows users to use standard programming languages like $\mathrm{C}++$ and Python. A low level version of ROS is also available for the Raspberry $\mathrm{Pi}$, enabling robot control using standard ROS nodes as well as transmission of data to other PCs via a remote Roscore host.

Multiple control schemes are planned for the use of this robot, one being the classical method by planning and executing motions on board.

\section{A. Pantograph Leg Design}

The use of a pantograph dates back to at least 1605 as a tool to copy and scale diagrams [24]. As a mechanical design for legs it has been proposed for leg prosthesis [25] as well as robotic legs [26] and consequently quadruped robots like the Cheetah-Cub [27]. Coupled with a springmass system a pantograph leg can be created to behave close to an animals leg [28]. The design of the legs for this work as described in [23] used such a pantograph. This way the leg can be actuated to perform a step sequence, lifting the toes high enough as to not collide with the ground or small obstacles, without direct actuation of the ankle. A Spring was implemented to stretch the knee, allowing for a tendon driven knee joint, while holding the weight of the robot. A second spring was used to introduce elasticity into the ankle joint, to enable the foot to adapt to different surface structures on the ground (Figure 1 top).

The goal of the legs in version 2.1 was to reduce the complexity of the legs to a single piece $3 \mathrm{D}$ printed leg, replacing the joints with flexible hinges. This way however, the needed pantograph design could not be recreated.

The newest design of the leg implements the original pantograph design, while using the flexible hinges created for version 2.1 and keeping the complexity low with only two functional parts. The legs are pictured in Figure 6. The two parts are the leg itself and a "spacer" connecting the femur with the ankle, which can be "snapped" into the leg. The spacer can be pivoted between its two bearing points, extending the ankle joint together with the flexing of the knee. This way, when the knee is bend, the angle between the tibia and the foot is reduced, lifting the toes to prevent any collisions with the ground.

The initially used spring used for the adaptation of the foot to the ground is introduced by an additional flexible hinge after the ankle, which is decoupled from the pantograph functionality. This way, during stance phase, the foot is kept as flat on the ground as possible, to create the necessary friction for a forward motion of the robot.

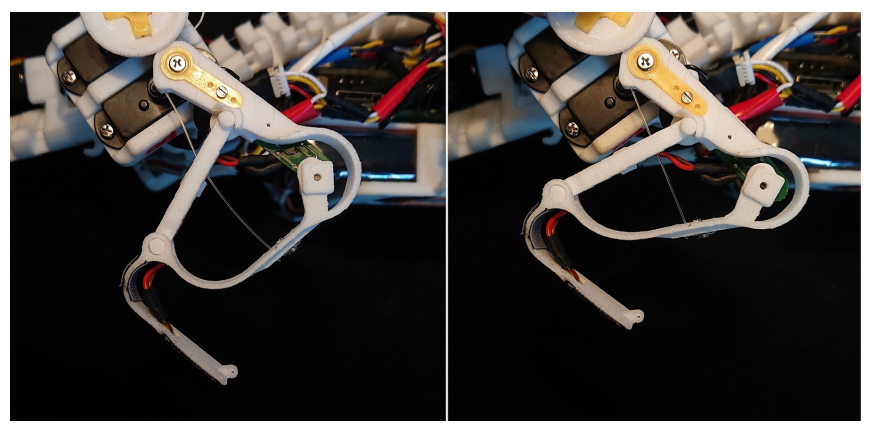

Fig. 6. Right Hindleg with a relaxed (left) and bend knee (right). The angle between the foot and the femur changes according to the knee angle, lifting the toes up during the swing phase of the leg.

\section{B. Comparison}

The following table (Table II) shows a comparison of the different robot versions as well as some earlier rodent-like and small quadruped robots. In the latest version, the weight increased due to the new electronics (which also needed a bigger battery) and the much heavier head. The length increase is due to the longer tail.

Other comparable robots are the Waseda Rats (WR), which 
are also representations of a rat in robot form. However, starting with the 3rd version Shi et al. used wheeled robots, to create the needed speed for their research. The Waseda Rats are measured in length without their tails, when done so with the NeRmo it has a length of $227 \mathrm{~mm}$, which is shorter than the Waseda Rats. They are smaller in width, but also heavier due to their metal construction. The speed of WR-1 and -2 could be surpassed by the NeRmo, but not for the wheeled version five. Other comparable small quadrupeds are the Cheeta-Cubs from the Biorobotics Laboratory at EPFL. Those Robots are generally higher and heavier than the NeRmo but also faster. Only the Speed of the Cheetah-CubS could almost be reached.

TABLE II

NERMO IN COMPARISON TO ITSELF, OTHER SIMILAR ROBOTS AND rattus norvegicus. DIMENSIONS ARE LENGTH X WIDTH X HEIGHT.

\begin{tabular}{|l|c|c|c|}
\hline Robot & Dimension $(\mathrm{mm})$ & Weight $(\mathrm{kg})$ & Speed $(\mathrm{m} / \mathrm{s})$ \\
\hline NeRmo V4.0 & $405 \times 91 \times 90$ & 0.265 & 0.3 \\
NeRmo V3.0 & $310 \times 81 \times 85$ & 0.181 & 0.1 \\
NeRmo V2.1 & $340 \times 85 \times 70.6$ & 0.148 & 0.1 \\
NeRmo V2.0 & $340 \times 85 \times 70.6$ & 0.200 & 0.1 \\
NeRmo V1.0 & $316 \times 72 \times 85$ & 0.225 & 0.06 \\
\hline Cheetah-Cub [29] & $210 \times 100 \times 158$ & 1.10 & 1.42 \\
Cheetah-CubS [29] & $205 \times 100 \times 105$ & 1.16 & 0.36 \\
WR-1 [30] & $270 \times 130 \times 110$ & 1.15 & 0.02 \\
WR-2 [30] & $240 \times 70 \times 90$ & 0.85 & 0.03 \\
WR-5 [30], [31] & 2407090 & 0.70 & 1.0 \\
\hline rattus norvegicus[32] & length 370-440 & $0.25-0.4$ & $0.1-0.8$ \\
\hline
\end{tabular}

\section{OUTLOOK}

We could show that the necessary requirements as described in the introduction could can be met by the here presented robot. In the future we plan to use this robot together with its digital twin inside the Neurorobotics Platform (NRP), an embodied simulation environment developed within the Human Brain Project, to further research on neural control of movement in small animals. This entails generating motions from animal as described in [33] and implementing brain derived spiking neural networks, capable of learning simple motions up to learning different gaits. NeRmo has unique features as a hybrid soft-hard robot. Through dynamic simulations with NRP, we will improve the geometries of NeRmo, especially in terms of the flexible spine functionality. Comparative analysis between the virtual NeRmo and the mouse musculoskeletal mode is also our scope. We will train and refine these controllers on the NRP, and eventually transfer them onto the physical robot.

\section{ACKNOWLEDGMENT}

We appreciate Dr. Fabrice Morin for his insightful advice which greatly improved the manuscript.

\section{REFERENCES}

[1] R. Lai, W. Lin, and Y. Wu, "Review of research on the key technologies, application fields and development trends of intelligent robots," in Intelligent Robotics and Applications, Z. Chen, A. Mendes, Y. Yan, and S. Chen, Eds., Cham: Springer International Publishing, 2018, pp. 449-458, ISBN: 978-3-319-97589-4.

[2] P. Fankhauser. (2018). Worlds first autonomous offshore robot, [Online]. Available: https : / / wWw . anybotics . com/2018/10/25/worlds first-autonomous-offshore-robot/ (visited on 07/05/2019).

[3] E. Ackerman. (2018). Boston dynamics is getting ready to produce lots of spotminis, [Online]. Available: https : / / spectrum . ieee. org / automaton / robotics / industrial robots/boston-dynamics-spotminis (visited on 07/05/2019).

[4] A. Takanishi, T. Aoki, M. Ito, Y. Ohkawa, and J. Yamaguchi, "Interaction between creature and robot: Development of an experiment system for rat and rat robot interaction," in Proc. IEEE/RSJ Int. Conf. Intelligent Robots and Systems. Innovations in Theory No.98CH36190) Practice and Applications (Cat, Robotics - Rat, IEEE, vol. 3, Oct. 1998, pp. 19751980. DOI: 10.1109/IROS.1998.724896. [Online]. Available: http : / / ieeexplore. ieee. org/document/724896/.

[5] F. Patanè, V. Mattoli, C. Laschi, B. Mazzolai, P. Dario, H. Ishii, and A. Takanishi, "Biomechatronic design and development of a legged rat robot," in Robotics and Biomimetics, 2007. ROBIO 2007. IEEE International Conference on, Robotics - Rat, IEEE, 2007, pp. 847-852.

[6] H. Yang, T. A. Bell, G. A. Churchill, and F. P.-M. De Villena, "On the subspecific origin of the laboratory mouse," Nature genetics, vol. 39, no. 9, p. 1100, 2007.

[7] S. Oota, Y. Okamura-Oho, K. Ayusawa, Y. Ikegami, A. Murai, E. Yoshida, and Y. Nakamura, "Neurorobotic approach to study huntington disease based on a mouse neuromusculoskeletal model," 2018 IEEE/RSJ International Conference on Intelligent Robots and Systems (IROS), pp. 6720-6727, 2018.

[8] L. M. Klyatis and E. L. Klyatis, "Chapter 1 - accurate physical simulation of field input influences on the actual product," in Accelerated Quality and Reliability Solutions, L. M. Klyatis and E. L. Klyatis, Eds., Oxford: Elsevier Science, 2006, pp. 192, ISBN: 978-0-08-044924-1. DOI: https : / / doi . org / 10 . 1016/B978-008044924-1/ $50002-2$. [Online]. Available: http : / / www . sciencedirect . com / science / article / pii/B9780080449241500022.

[9] R. Bish, S. Joshi, J. Schank, and J. Wexler, "Mathematical modeling and computer simulation of a 
robotic rat pup," Mathematical and computer modelling, vol. 45, no. 7-8, pp. 981-1000, 2007.

[10] S. Ivaldi, V. Padois, and F. Nori, "Tools for dynamics simulation of robots: A survey based on user feedback," arXiv preprint arXiv:1402.7050, 2014.

[11] T. Lens, K. Radkhah, and O. von Stryk, "Simulation of dynamics and realistic contact forces for manipulators and legged robots with high joint elasticity," in 2011 15th International Conference on Advanced Robotics (ICAR), IEEE, 2011, pp. 34-41.

[12] K. Karakasiliotis, R. Thandiackal, K. Melo, T. Horvat, N. K. Mahabadi, S. Tsitkov, J.-M. Cabelguen, and A. J. Ijspeert, "From cineradiography to biorobots: An approach for designing robots to emulate and study animal locomotion," Journal of The Royal Society Interface, vol. 13, no. 119, p. 20151 089, 2016.

[13] B. Wang, X. Zou, and J. Zhu, "Data assimilation and its applications," Proceedings of the National Academy of Sciences, vol. 97, no. 21, pp. 11 143-11 144, 2000.

[14] K. E. Jones, L. Benitez, K. D. Angielczyk, and S. E. Pierce, "Adaptation and constraint in the evolution of the mammalian backbone," BMC evolutionary biology, vol. 18, no. 1, p. 172, 2018.

[15] L. M. Frolich and A. A. Biewener, "Kinematic and electromyographic analysis of the functional role of the body axis during terrestrial and aquatic locomotion in the salamander ambystoma tigrinum," Journal of Experimental Biology, vol. 162, no. 1, pp. 107-130, 1992.

[16] M. A. Ashley-Ross and B. F. Bechtel, "Kinematics of the transition between aquatic and terrestrial locomotion in the newt taricha torosa," Journal of Experimental Biology, vol. 207, no. 3, pp. 461-474, 2004.

[17] C. M. Bagi, E. Berryman, and M. R. Moalli, "Comparative bone anatomy of commonly used laboratory animals: Implications for drug discovery," Comparative medicine, vol. 61, no. 1, pp. 76-85, 2011.

[18] X. Hu, J. P. Charles, T. Akay, J. R. Hutchinson, and S. S. Blemker, "Are mice good models for human neuromuscular disease? comparing muscle excursions in walking between mice and humans," Skeletal muscle, vol. 7, no. 1, p. 26, 2017.

[19] F. Yue, Y. Cheng, A. Breschi, J. Vierstra, W. Wu, T. Ryba, R. Sandstrom, Z. Ma, C. Davis, B. D. Pope, et al., "A comparative encyclopedia of dna elements in the mouse genome," Nature, vol. 515, no. 7527, p. 355, 2014.

[20] M. I. Siegel, "The tail, locomotion and balance in mice," American Journal of Physical Anthropology, vol. 33, no. 1, pp. 101-102, 1970.

[21] H. SHINOHARA, "The musculature of the mouse tail is characterized by metameric arrarmements of bicipital muscles," Okajimas folia anatomica Japonica, vol. 76, no. 4, pp. 157-169, 1999.
[22] B. A. Radvansky and D. A. Dombeck, "An olfactory virtual reality system for mice," Nature communications, vol. 9, no. 1, p. 839, 2018.

[23] P. Lucas and A. K. Florian Walter, "Design of a biomimetic rodent robot," Technische Universitaet Muenchen, Institut fuer Informatik, Tech. Rep. TUMI1873, 2018.

[24] C. SCHEINER, Christophori Scheiner ... Pantographice, Seu Ars Delineandi Res Quaslibet Per Parallelogrammum Lineare Seu Cavum Mechanicum Mobile, Libellis Duobus Explicata, Etc. Ex typographia L. Grignani, 1631. [Online]. Available: https : / / books . google . de / books ? id = dETPMgEACAAJ.

[25] U. K. Henschke and H. A. Mauch, Leg prosthesis, US Patent 2,489,291, Nov. 1949.

[26] C. Liang, M. Ceccarelli, and Y. Takeda, "Operation analysis of a chebyshev-pantograph leg mechanism for a single dof biped robot," Frontiers of Mechanical Engineering, vol. 7, Dec. 2012. DOI: 10 . 1007 / s11465-012-0340-5.

[27] A. Sproewitz, M. Fremerey, K. Karakasiliotis, S. Rutishauser, L. Righetti, and A. J. Ijspeert, "Compliant leg design for a quadruped robot," in Abstracts of Dynamic Walking 2009, Robotics - Bioinspired, 2009.

[28] H. Witte, R. Hackert, W. Ilg, J. Biltzinger, N. Schilling, F. Biedermann, M. Jergas, H. Preuschoft, M. S. Fischer, F. Informatik, I. D.-. U. Servicesysteme, and T. Karlsruhe, "Quadrupedal mammals as paragons for walking machines," in Proceeding of the International Symposium on Adaptive Motion in Animals and Machines (AMAM, 2000, pp. 8-12.

[29] E. Biorobotics Laboratory BIOROB. (2017). Quadruped robotics, [Online]. Available: http : / / biorob. epfl. ch / research / quadruped (visited on 06/21/2017).

[30] Q. Shi. (2014). Mental modeling of creature. Robotics - Web, Takanishi Laboratory at Waseda University, [Online]. Available: http://www . takanishi . mech. waseda.ac.jp/top/research/rat / robot-WR.html (visited on 05/24/2017).

[31] Q. Shi, H. Ishii, H. Sugita, S. Kinoshita, Z. Lin, A. Takanishi, S. Okabayashi, N. Iida, and H. Kimura, "A rat-like robot wr-5 for animal behavior research," in 2012 IEEE International Conference on Robotics and Biomimetics (ROBIO), Dec. 2012, pp. 784-789. DOI: 10.1109/ROBIO.2012.6491063.

[32] M. S. Fischer, N. Schilling, M. Schmidt, D. Haarhaus, and H. Witte, "Basic limb kinematics of small therian mammals," Journal of Experimental Biology, vol. 205, no. 9, pp. 1315-1338, 2002, Biology - Rat.

[33] K. Ayusawa, Y. Ikegami, A. Murai, Y. Yoshiyasu, E. Yoshida, S. Oota, and Y. Nakamura, "Interspecies retargeting of homologous body posture based on skeletal morphing," 2018 IEEE/RSJ International Conference on Intelligent Robots and Systems (IROS), pp. 6712-6719, 2018. 